\title{
A Study on the Class of Chain Ratio-type Estimators
}

\author{
Housila P. Singh and Anjana Rathour*
}

Vikram University

\begin{abstract}
This paper considers the problem of estimating the population mean $\bar{Y}$ of the study variate $y$ using information on different parameters such as population mean $(\bar{X})$, coefficient of variation $\left(C_{x}\right)$, kurtosis $\left(\beta_{2(x)}\right)$, standard deviation $\left(S_{x}\right)$ of the auxiliary variate $x$ and on the correlation coefficient, $\rho$, between the study variate $y$ and the auxiliary variate $x$ through transformation. A class of estimators on the lines of Kadilar and Cingi (2003) has been defined and its properties are studied to the first degree of approximation. It has been shown that the proposed class of estimators is better than usual unbiased estimator $\bar{y}$, ratio estimator $\bar{y}_{R}$, ratio-type estimator $t_{R}$ and Kadilar and Cingi (2003) estimator $\bar{y}_{C R}$ under some realistic conditions. Numerical illustration is given in support of the present study.
\end{abstract}

Keywords. Study variate; auxiliary variate; simple random sampling; ratio estimator; Chain ratio-type estimator.

MSC 2010: 62D05.

\section{Introduction}

Consider a finite population $U=\left\{U_{1}, U_{2}, \ldots, U_{N}\right\}$ of size $N$. Let $y$ and $x$ be the study variate and auxiliary variate, taking values $y_{i}$ and $x_{i}$ respectively for the $i$ th unit $U_{i}(i=1,2, \ldots, N)$. The parameter of interest is the population mean $\bar{Y}$ of the study variate $y$. The precision of estimators of the population mean $\bar{Y}$ can be increased by utilizing advance information about

* Corresponding author 
a suitable auxiliary variable $x$ correlated with $y$. Out of many ratio, product, difference and regression methods of estimation are good examples in this context. Let $\bar{X}$ be the known population mean of the auxiliary variate $x$ and $\bar{y}, \bar{x}$ respectively denote the sample means of the variates $y$ and $x$ based on a simple random sample of size $n$ drawn without replacement from the population $U$. Also let:

$$
\begin{gathered}
S_{y}^{2}=(N-1)^{-1} \sum_{j=1}^{N}\left(y_{j}-\bar{Y}\right)^{2}, \quad S_{x}^{2}=(N-1)^{-1} \sum_{j=1}^{N}\left(x_{j}-\bar{X}\right)^{2}, \quad f=\frac{n}{N} \\
R=\frac{\bar{Y}}{\bar{X}}, \quad S_{x y}=(N-1)^{-1} \sum_{j=1}^{N}\left(x_{j}-\bar{X}\right)\left(y_{j}-\bar{Y}\right), \quad C_{y}^{2}=\frac{S_{y}^{2}}{\bar{Y}^{2}}, \quad C_{x}^{2}=\frac{S_{x}^{2}}{\bar{X}^{2}}
\end{gathered}
$$

and $\rho=S_{x y} /\left(S_{x} S_{y}\right)$ denotes the correlation coefficient between the study variate $y$ and the auxiliary variate $x$.

The classical ratio estimator for the population mean $\bar{Y}$ of the study variate $y$ is defined by

$$
\bar{y}_{R}=\bar{y}\left(\frac{\bar{X}}{\bar{x}}\right) \text {, }
$$

where it is assumed that the population mean $\bar{X}$ of the auxiliary variate $x$ is known.

Replacing $\bar{y}$ by $\bar{y}_{R}$ in (1), Kadilar and Cingi (2003) suggested the chain ratio-type estimator for population mean $\bar{Y}$ as

$$
\bar{y}_{C R}=\bar{y}_{R}\left(\frac{\bar{X}}{\bar{x}}\right)=\bar{y}\left(\frac{\bar{X}^{2}}{\bar{x}^{2}}\right) .
$$

Kadilar and Cingi (2003) have shown that the chain ratio-type estimator $\bar{y}_{C R}$ is more efficient than the usual ratio estimator $\bar{y}_{R}$ if

$$
k\left(=\rho \frac{C_{y}}{C_{x}}\right)>\frac{3}{2} .
$$

It is observed in the sampling theory literature that in recent years considerable attempts have been made by various authors to increase precision of the conventional estimators by using suitable transformation on the auxiliary variate $x$, for instance, see Mohanty and Das (1971), Srivenkataramana (1978, 1980), Sisodia and Dwivedi (1981), Singh and Tailor (2003), Singh et al. (2004), Upadhyaya and Singh (1999) and Kadilar and Cingi (2006). Using the transformation

$$
z_{i}=\eta x_{i}+\lambda, \quad i=1,2, \ldots, N
$$


on the auxiliary variate $x$, a ratio-type estimator for population mean $\bar{Y}$ is defined by

$$
t_{R}=\bar{y} \frac{\bar{Z}}{\bar{z}}=\bar{y}\left(\frac{\eta \bar{X}+\lambda}{\eta \bar{x}+\lambda}\right)
$$

where $\bar{z}=(\eta \bar{x}+\lambda), \bar{Z}=(\eta \bar{X}+\lambda)$ and $\eta(\neq 0), \lambda$ are either real number or function of the known parameters of the auxiliary variate $x$ such as the standard deviation $\left(S_{x}\right)$, coefficient of variation $\left(C_{x}\right)$, skewness $\left\{\beta_{1}(x)\right\}$, kurtosis $\left\{\beta_{2}(x)\right\}, \Delta=\left(\beta_{2}(x)-\beta_{1}(x)-1\right)$ and the correlation coefficient $(\rho)$ of the population, see Singh and Agnihotri (2008) and Tripathi and Singh (1992). Some members of $t_{R}$ are listed in Table 1.

It is well known under simple random sampling without replacement (SRSWOR) that the variance/mean squared error (MSE) of the sample mean $\bar{y}$ is given by

$$
\operatorname{var}(\bar{y})=\operatorname{MSE}(\bar{y})=\frac{(1-f)}{n} S_{y}^{2}=\frac{(1-f)}{n} \bar{Y}^{2} C_{y}^{2}
$$

To the first degree of approximation, the mean squared error of the estimators $\bar{y}_{R}, \bar{y}_{C R}$ and $t_{R}$ are respectively given by

$$
\begin{gathered}
\operatorname{MSE}\left(\bar{y}_{R}\right)=\frac{(1-f)}{n}\left(S_{y}^{2}+R^{2} S_{x}^{2}-2 R \rho S_{x} S_{y}\right) \\
\operatorname{MSE}\left(\bar{y}_{C R}\right)=\frac{(1-f)}{n}\left(S_{y}^{2}+4 R^{2} S_{x}^{2}-4 R \rho S_{x} S_{y}\right) \\
\operatorname{MSE}\left(t_{R}\right)=\frac{(1-f)}{n}\left(S_{y}^{2}+R^{*^{2}} S_{x}^{2} \eta^{2}-2 \eta R^{*} \rho S_{x} S_{y}\right)
\end{gathered}
$$

where $R^{*}=\bar{Y} /(\eta \bar{X}+\lambda)$.

The plan of this article is as follows. The first section is the introduction. The class of chain ratio-type estimators $t_{C R}$ has been proposed with its bias and MSE expressions in Section 2. In Section 3, we have presented the efficiency comparisons of the proposed estimator $t_{C R}$ with estimators $\bar{y}, \bar{y}_{R}$, $\bar{y}_{C R}$ and $t_{R}$. An empirical study is carried out in Section 4 to judge the merits of the suggested class of chain ratio-type estimators $t_{C R}$ over other estimators. 


\section{The Class of Chain Ratio-type Estimators}

When $\bar{y}$ in (4) is replaced with $t_{R}$, the class of chain ratio-type estimators is obtained as

$$
t_{C R}=\frac{t_{R}(\eta \bar{X}+\lambda)}{\eta \bar{x}+\lambda},
$$

we can re-write (9) using (4) as

$$
t_{C R}=\frac{\bar{y}(\eta \bar{X}+\lambda)^{2}}{(\eta \bar{x}+\lambda)^{2}},
$$

which may be further generalized as

$$
t_{C R(\alpha)}=\bar{y}\left(\frac{\eta \bar{X}+\lambda}{\eta \bar{x}+\lambda}\right)^{\alpha}
$$

where $\alpha$ is a suitably chosen constant, see Srivastava (1967). The optimum value of $\alpha$ is $k\{1+\lambda /(\eta \bar{X})\}$. Rao (1991) calls this generalized form of the estimator the repeated substitution estimator. Some members of the estimator $t_{C R}$ are listed in Table 2.

The MSE of the class of chain ratio-type estimator can be obtained using the Taylor series expansion. In general, this expansion for a function of $p$ variables can be given as

$$
h\left(\bar{x}_{1}, \bar{x}_{2}, \ldots, \bar{x}_{p}\right)=h\left(\bar{X}_{1}, \bar{X}_{2}, \ldots, \bar{X}_{p}\right)+\sum_{j=1}^{p} d_{j}\left(\bar{x}_{j}-\bar{X}_{j}\right)+R_{p}\left(\bar{X}_{p}, a\right)
$$

where

$$
d_{j}=\frac{\partial h\left(a_{1}, a_{2}, \ldots, a_{p}\right)}{\partial a_{j}}
$$

and

$$
R_{p}\left(\bar{X}_{p}, a\right)=\sum_{j=1}^{p} \sum_{i=1}^{p} \frac{1}{2 !} \cdot \frac{\partial^{2} h\left(\bar{X}_{1}, \bar{X}_{2}, \ldots, \bar{X}_{p}\right)}{\partial \bar{X}_{i} \partial \bar{X}_{j}}\left(\bar{x}_{j}-\bar{X}_{j}\right)\left(\bar{x}_{i}-\bar{X}_{i}\right)+O_{p}
$$

where $O_{p}$ denotes the remainder of the Taylor series expansion having terms of degree higher than two, see Wolter (1985). Omitted the term $R_{p}\left(\bar{X}_{p}, a\right)$, we obtain the Taylor series method for two variables as

$$
h(\bar{x}, \bar{y})-\left.h(\bar{X}, \bar{Y}) \cong \frac{\partial h(c, d)}{\partial c}\right|_{(\bar{X}, \bar{Y})}(\bar{x}-\bar{X})+\left.\frac{\partial h(c, d)}{\partial d}\right|_{(\bar{X}, \bar{Y})}(\bar{y}-\bar{Y})
$$


see Lohr (1999). Here

$$
h(\bar{x}, \bar{y})=\frac{\bar{y}}{(\eta \bar{x}+\lambda)^{2}}=\hat{R}_{c} \quad \text { and } \quad h(\bar{X}, \bar{Y})=\frac{\bar{Y}}{(\eta \bar{X}+\lambda)^{2}}=R_{c} .
$$

From (12) and (13) we can write

$$
\hat{R}_{c}-R_{c} \cong-\frac{2 \bar{Y} \eta(\eta \bar{X}+\lambda)}{(\eta \bar{X}+\lambda)^{4}}(\bar{x}-\bar{X})+\frac{1}{(\eta \bar{X}+\lambda)^{2}}(\bar{y}-\bar{Y}) .
$$

Squaring both sides of (14) we have

$$
\begin{aligned}
\left(\hat{R}_{c}-R_{c}\right)^{2} \cong & \frac{4 \bar{Y}^{2} \eta^{2}(\eta \bar{X}+\lambda)^{2}}{(\eta \bar{X}+\lambda)^{8}}(\bar{x}-\bar{X})^{2}-\frac{4 \bar{Y} \eta(\eta \bar{X}+\lambda)}{(\eta \bar{X}+\lambda)^{6}}(\bar{x}-\bar{X})(\bar{y}-\bar{Y}) \\
& +\frac{1}{(\eta \bar{X}+\lambda)^{4}}(\bar{y}-\bar{Y})^{2}
\end{aligned}
$$

or

$$
\left(\hat{R}_{c}-R_{c}\right)^{2} \cong \frac{1}{(\eta \bar{X}+\lambda)^{4}}\left\{4 \eta^{2} R^{*^{2}}(\bar{x}-\bar{X})^{2}-4 \eta R^{*}(\bar{x}-\bar{X})(\bar{y}-\bar{Y})+(\bar{y}-\bar{Y})^{2}\right\} .
$$

Taking expectation of both sides of (15) we get

$$
\begin{aligned}
E\left(\hat{R}_{c}-R_{c}\right)^{2} & \cong \frac{1}{(\eta \bar{X}+\lambda)^{4}}\left\{4 \eta^{2} R^{*^{2}} V(\bar{x})-4 \eta R^{*} \operatorname{cov}(\bar{x}, \bar{y})+V(\bar{y})\right\} \\
& =\frac{(1-f)}{n(\eta \bar{X}+\lambda)^{4}}\left\{S_{y}^{2}+4 \eta^{2} R^{*^{2}} S_{x}^{2}-4 \eta R^{*} \rho S_{x} S_{y}\right\} .
\end{aligned}
$$

Thus the MSE of to the first degree of approximation is given by

$$
\operatorname{MSE}\left(t_{C R}\right)=\frac{(1-f)}{n}\left(S_{y}^{2}+4 \eta^{2} R^{*^{2}} S_{x}^{2}-4 \eta R^{*} \rho S_{x} S_{y}\right)
$$

The bias of $t_{C R}$ is given by

$$
\begin{aligned}
B\left(t_{C R}\right) & =E\left(t_{C R}-\bar{Y}\right) \\
& =E\left\{\hat{R}_{c}(\eta \bar{X}+\lambda)^{2}-R_{c}(\eta \bar{X}+\lambda)^{2}\right\} \\
& =(\eta \bar{X}+\lambda)^{2} E\left(\hat{R}_{c}-R_{c}\right) \\
& =(\eta \bar{X}+\lambda)^{2} B\left(\hat{R}_{c}\right)
\end{aligned}
$$


where $B\left(t_{C R}\right)$ stands for the bias of $\hat{R}_{c}$. To the first degree of approximation, the bias of $\hat{R}_{c}$ is obtained as follows:

$$
\begin{aligned}
\hat{R}_{c}-R_{c}= & h(\bar{x}, \bar{y})-h(\bar{X}, \bar{Y}) \\
= & {\left[\left.\frac{\partial h(c, d)}{\partial c}\right|_{(\bar{X}, \bar{Y})}(\bar{x}-\bar{X})+\left.\frac{\partial h(c, d)}{\partial d}\right|_{(\bar{X}, \bar{Y})}(\bar{y}-\bar{Y})\right.} \\
& +\frac{1}{2 !}\left\{\left.\frac{\partial^{2} h(c, d)}{\partial c^{2}}\right|_{(\bar{X}, \bar{Y})}(\bar{x}-\bar{X})^{2}\right. \\
& \left.\left.+\left.2 \frac{\partial^{2} h(c, d)}{\partial d \partial c}\right|_{(\bar{X}, \bar{Y})}(\bar{x}-\bar{X})(\bar{y}-\bar{Y})+\left.\frac{\partial^{2} h(c, d)}{\partial d^{2}}\right|_{(\bar{X}, \bar{Y})}(\bar{y}-\bar{Y})^{2}\right\}\right] .
\end{aligned}
$$

We note that

$$
\begin{gathered}
\left.\frac{\partial h(c, d)}{\partial c}\right|_{(\bar{X}, \bar{Y})}=\frac{-2 \eta \bar{Y}}{(\eta \bar{X}+\lambda)^{3}}, \\
\left.\frac{\partial h(c, d)}{\partial d}\right|_{(\bar{X}, \bar{Y})}=\frac{1}{(\eta \bar{X}+\lambda)^{2}}, \\
\left.\frac{\partial^{2} h(c, d)}{\partial c^{2}}\right|_{(\bar{X}, \bar{Y})}=\frac{-6 \eta^{2} \bar{Y}}{(\eta \bar{X}+\lambda)^{4}}, \\
\left.\frac{\partial^{2} h(c, d)}{\partial d^{2}}\right|_{(\bar{X}, \bar{Y})}=0,
\end{gathered}
$$

and

$$
\left.\frac{\partial^{2} h(c, d)}{\partial d \partial c}\right|_{(\bar{X}, \bar{Y})}=\frac{-2 \eta}{(\eta \bar{X}+\lambda)^{3}} .
$$

Putting (19)-(23) in (18) we have

$$
\begin{aligned}
\left(\hat{R}_{c}-R_{c}\right)= & -\frac{2 \eta \bar{Y}}{(\eta \bar{X}+\lambda)^{3}}(\bar{x}-\bar{X})+\frac{1}{(\eta \bar{X}+\lambda)^{2}}(\bar{y}-\bar{Y})+\frac{1}{2}\left\{\frac{6 \eta^{2} \bar{Y}}{(\eta \bar{X}+\lambda)^{2}}\right. \\
& \left.\times(\bar{x}-\bar{X})^{2}-\frac{4 \eta}{(\eta \bar{X}+\lambda)^{3}}(\bar{x}-\bar{X})(\bar{y}-\bar{Y})\right\} .
\end{aligned}
$$

Taking expectation of both sides of $(24)$ we get the bias of $\hat{R}_{c}$ to the first degree of approximation as

$$
B\left(\hat{R}_{c}\right)=\frac{(1-f)}{n} \cdot \frac{\eta}{(\eta \bar{X}+\lambda)^{3}}\left(3 \eta R^{*} S_{x}^{2}-2 \rho S_{x} S_{y}\right)
$$


Substitution of (25) in (17) yields the bias of $t_{C R}$ to the first degree of approximation as

$$
B\left(t_{C R}\right)=\frac{(1-f)}{n} \cdot \frac{\eta}{(\eta \bar{X}+\lambda)}\left(3 \eta R^{*} S_{x}^{2}-2 \rho S_{x} S_{y}\right) .
$$

The bias of $t_{C R}$ will vanish either (i) the sample size $\mathrm{n}$ is large enough, or (ii) $k=3 / 2\{1+\lambda /(\eta \bar{X})\}^{-1}$.

The bias term is omitted for the first degree of approximation in the Taylor series expanssion while obtaining the MSE of the proposed estimator $T_{C R}$.

\section{Efficiency Comparison}

From (5), (6), (7), (8) and (16) we have

(i)

$$
\begin{aligned}
\operatorname{MSE}(\bar{y})-\operatorname{MSE}\left(t_{C R}\right) & =\frac{4(1-f)}{n} \eta R^{*} S_{x}^{2}\left(\beta-\eta R^{*}\right) \\
& =\frac{4(1-f)}{n} \eta R R^{*} S_{x}^{2}(k-\theta)>0 \text { if } \quad \theta<k,
\end{aligned}
$$

where $\theta=\eta \bar{X} /(\eta \bar{X}+\lambda)$ and $k=\beta / R$.

(ii) $\operatorname{MSE}\left(\bar{y}_{R}\right)-\operatorname{MSE}\left(t_{C R}\right)=\frac{(1-f)}{n}(1-2 \theta)(1+2 \theta-2 k) R^{2} S_{x}^{2}>0$ if

$$
\left.\begin{array}{lr}
\text { either } & \frac{1}{2}<\theta<\frac{1}{2}(2 k-1) \\
\text { or } & \frac{1}{2}(2 k-1)<\theta<\frac{1}{2}
\end{array}\right\}
$$

or equivalently,

$$
\min \left\{\frac{1}{2}, \frac{1}{2}(2 k-1)\right\}<\theta<\max \left\{\frac{1}{2}, \frac{1}{2}(2 k-1)\right\},
$$

(iii) $\operatorname{MSE}\left(\bar{y}_{C R}\right)-\operatorname{MSE}\left(t_{C R}\right)=\frac{4(1-f)}{n} R^{2} S_{x}^{2}(1-\theta)(1+\theta-k)>0$ if

$$
\left.\begin{array}{lc}
\text { either } & (k-1)<\theta<1 \\
\text { or } & 1<\theta<(k-1)
\end{array}\right\}
$$

or equivalently,

$$
\min \{1,(k-1)\}<\theta<\max \{1,(k-1)\}
$$


Table 1. Some members of the estimator $t_{R}$

\begin{tabular}{|c|c|c|c|c|c|}
\hline Estimator & $\eta$ & $\lambda$ & Estimator & $\eta$ & $\lambda$ \\
\hline$t_{R(1)}=\bar{y}\left(\frac{\bar{x}}{\bar{x}}\right)$ & 1 & 0 & $t_{R(12)}=\bar{y}\left(\frac{S_{x} \bar{X}+C_{x}}{S_{x} \bar{x}+C_{x}}\right)$ & $S_{x}$ & $C_{x}$ \\
\hline$t_{R(2)}=\bar{y}\left(\frac{\bar{X}+C_{x}}{\bar{x}+C_{x}}\right)$ & 1 & $C_{x}$ & $t_{R(13)}=\bar{y}\left(\frac{S_{x} \bar{X}+\beta_{2(x)}}{S_{x} \bar{x}+\beta_{2(x)}}\right)$ & $S_{x}$ & $\beta_{2(x)}$ \\
\hline$t_{R(3)}=\bar{y}\left(\frac{\bar{X}+\beta_{2(x)}}{\bar{x}+\beta_{2(x)}}\right)$ & 1 & $\beta_{2(x)}$ & $t_{R(14)}=\bar{y}\left(\frac{\bar{x} \beta_{2(x)}+S_{x}}{\overline{\bar{x}} \beta_{2(x)}+S_{x}}\right)$ & $\beta_{2(x)}$ & $S_{x}$ \\
\hline$t_{R(4)}=\bar{y}\left(\frac{\bar{x}+\rho}{\bar{x}+\rho}\right)$ & 1 & $\rho$ & $t_{R(15)}=\bar{y}\left(\frac{\bar{X} C_{x}+S_{x}}{\bar{x} C_{x}+S_{x}}\right)$ & $C_{x}$ & $S_{x}$ \\
\hline$t_{R(5)}=\bar{y}\left(\frac{\bar{X}+S_{x}}{\bar{x}+S_{x}}\right)$ & 1 & $S_{x}$ & $t_{R(16)}=\bar{y}\left(\frac{\bar{x} \rho+S_{x}}{\bar{x} \rho+S_{x}}\right)$ & $\rho$ & $S_{x}$ \\
\hline$t_{R(6)}=\bar{y}\left(\frac{\beta_{2(x)} \bar{X}+C_{x}}{\beta_{2(x)} \bar{x}+C_{x}}\right)$ & $\beta_{2(x)}$ & $C_{x}$ & $t_{R(17)}=\bar{y}\left(\frac{S_{x} \bar{X}+\rho}{S_{x} \bar{x}+\rho}\right)$ & $S_{x}$ & $\rho$ \\
\hline$t_{R(7)}=\bar{y}\left(\frac{\bar{x} C_{x}+\beta_{2(x)}}{\bar{x} C_{x}+\beta_{2(x)}}\right)$ & $C_{x}$ & $\beta_{2(x)}$ & $t_{R(18)}=\bar{y}\left(\frac{\bar{X}+X}{\bar{x}+X}\right)$ & 1 & $X$ \\
\hline$t_{R(8)}=\bar{y}\left(\frac{\rho \bar{X}+C_{x}}{\rho \bar{x}+C_{x}}\right)$ & $\rho$ & $C_{x}$ & $t_{R(19)}=\bar{y}\left(\frac{C_{x} \bar{X}+X}{C_{x} \bar{x}+X}\right)$ & $C_{x}$ & $X$ \\
\hline$t_{R(9)}=\bar{y}\left(\frac{\bar{X} C_{x}+\rho}{\bar{x} C_{x}+\rho}\right)$ & $C_{x}$ & $\rho$ & $t_{R(20)}=\bar{y}\left(\frac{\beta_{2(x)} \bar{X}+X}{\beta_{2(x)} \bar{x}+X}\right)$ & $\beta_{2(x)}$ & $X$ \\
\hline$t_{R(10)}=\bar{y}\left(\frac{\bar{X} \beta_{2(x)}+\rho}{\bar{x} \beta_{2(x)}+\rho}\right)$ & $\beta_{2(x)}$ & $\rho$ & $t_{R(21)}=\bar{y}\left(\frac{\rho \bar{X}+X}{\rho \bar{x}+X}\right)$ & $\rho$ & $X$ \\
\hline$t_{R(11)}=\bar{y}\left(\frac{\rho \bar{X}+\beta_{2(x)}}{\rho \bar{x}+\beta_{2(x)}}\right)$ & $\rho$ & $\beta_{2(x)}$ & & & \\
\hline
\end{tabular}

where $X(=N \bar{X})$ is the population total of the auxiliary variate $x$.

(iv)

$$
\operatorname{MSE}\left(t_{R}\right)-\operatorname{MSE}\left(t_{C R}\right)=\frac{(1-f)}{n} \theta R^{2} S_{x}^{2}(2 k-3 \theta)>0 \quad \text { if } \quad \frac{3}{2}<\frac{k}{\theta} .
$$

\section{Empirical Study}

In this section we have compared the performance of the estimators $t_{R}$ and $t_{C R}$ with the following data set.

Population I: [Source: Kadilar and Cingi (2006)]

The data consist of 106 villages in the Marmarian region of Turkey in 1999.

The variates are defined as

$y=$ The level of apple production.

$x=$ The number of apple trees ( 1 unit $=100$ trees $).$ 
Table 2. Some members of the chain ratio-type estimator $t_{C R}$

\begin{tabular}{|c|c|c|c|c|c|}
\hline Estimator & $\eta$ & $\lambda$ & Estimator & $\eta$ & $\lambda$ \\
\hline$t_{C R(1)}=\bar{y}\left(\frac{\bar{x}}{\bar{x}}\right)^{2}$ & 1 & 0 & $t_{C R(12)}=\bar{y}\left(\frac{S_{x} \bar{X}+C_{x}}{S_{x} \bar{x}+C_{x}}\right)^{2}$ & $S_{x}$ & $C_{x}$ \\
\hline$t_{C R(2)}=\bar{y}\left(\frac{\bar{X}+C_{x}}{\bar{x}+C_{x}}\right)^{2}$ & 1 & $C_{x}$ & $t_{C R(13)}=\bar{y}\left(\frac{S_{x} \bar{X}+\beta_{2(x)}}{S_{x} \bar{x}+\beta_{2(x)}}\right)^{2}$ & $S_{x}$ & $\beta_{2(x)}$ \\
\hline$t_{C R(3)}=\bar{y}\left(\frac{\bar{X}+\beta_{2(x)}}{\bar{x}+\beta_{2(x)}}\right)^{2}$ & 1 & $\beta_{2(x)}$ & $t_{C R(14)}=\bar{y}\left(\frac{\bar{X} \beta_{2(x)}+S_{x}}{\bar{x} \beta_{2(x)}+S_{x}}\right)^{2}$ & $\beta_{2(x)}$ & $S_{x}$ \\
\hline$t_{C R(4)}=\bar{y}\left(\frac{\bar{X}+\rho}{\bar{x}+\rho}\right)^{2}$ & 1 & $\rho$ & $t_{C R(15)}=\bar{y}\left(\frac{\bar{X} C_{x}+S_{x}}{\bar{x} C_{x}+S_{x}}\right)^{2}$ & $C_{x}$ & $S_{x}$ \\
\hline$t_{C R(5)}=\bar{y}\left(\frac{\bar{X}+S_{x}}{\bar{x}+S_{x}}\right)^{2}$ & 1 & $S_{x}$ & $t_{C R(16)}=\bar{y}\left(\frac{\bar{X} \rho+S_{x}}{\bar{x} \rho+S_{x}}\right)^{2}$ & $\rho$ & $S_{x}$ \\
\hline$t_{C R(6)}=\bar{y}\left(\frac{\beta_{2(x)} \bar{X}+C_{x}}{\beta_{2(x)} \bar{x}+C_{x}}\right)^{2}$ & $\beta_{2(x)}$ & $C_{x}$ & $t_{C R(17)}=\bar{y}\left(\frac{S_{x} \bar{X}+\rho}{S_{x} \bar{x}+\rho}\right)^{2}$ & $S_{x}$ & $\rho$ \\
\hline$t_{C R(7)}=\bar{y}\left(\frac{\bar{X} C_{x}+\beta_{2(x)}}{\bar{x} C_{x}+\beta_{2(x)}}\right)^{2}$ & $C_{x}$ & $\beta_{2(x)}$ & $t_{C R(18)}=\bar{y}\left(\frac{\bar{X}+X}{\bar{x}+X}\right)^{2}$ & 1 & $X$ \\
\hline$t_{C R(8)}=\bar{y}\left(\frac{\rho \bar{X}+C_{x}}{\rho \bar{x}+C_{x}}\right)^{2}$ & $\rho$ & $C_{x}$ & $t_{C R(19)}=\bar{y}\left(\frac{C_{x} \bar{X}+X}{C_{x} \bar{x}+X}\right)^{2}$ & $C_{x}$ & $X$ \\
\hline$t_{C R(9)}=\bar{y}\left(\frac{\bar{X} C_{x}+\rho}{\bar{x} C_{x}+\rho}\right)^{2}$ & $C_{x}$ & $\rho$ & $t_{C R(20)}=\bar{y}\left(\frac{\beta_{2(x)} \bar{X}+X}{\beta_{2(x)} \bar{x}+X}\right)^{2}$ & $\beta_{2}(x)$ & $X$ \\
\hline$t_{C R(10)}=\bar{y}\left(\frac{\bar{X} \beta_{2(x)}+\rho}{\bar{x} \beta_{2(x)}+\rho}\right)^{2}$ & $\beta_{2(x)}$ & $\rho$ & $t_{C R(21)}=\bar{y}\left(\frac{\rho \bar{X}+X}{\rho \bar{x}+X}\right)^{2}$ & $\rho$ & $X$ \\
\hline$t_{C R(11)}=\bar{y}\left(\frac{\rho \bar{X}+\beta_{2(x)}}{\rho \bar{x}+\beta_{2(x)}}\right)^{2}$ & $\rho$ & $\beta_{2(x)}$ & & & \\
\hline
\end{tabular}

The required values of the parameters are

$$
\begin{gathered}
N=106, \quad n=20, \quad \bar{Y}=15.37, \quad \bar{X}=243.76, \quad S_{x}=491.89, \quad S_{y}=64.25, \\
\rho=0.82, \quad \beta_{2(x)}=25.71, \quad k=1.70 .
\end{gathered}
$$

We have computed the percent relative efficiency (PRE) of the estimators $t_{R(i)}$ and $t_{C R(i)}$, ( $\mathrm{i}=1$ to 21 ) with respect to usual unbiased estimator $\bar{y}$ by using the following formulas

$\operatorname{PRE}\left(t_{R(i)}, \bar{y}\right)=\left\{1+R_{(i)}^{2}\left(\frac{S_{x}^{2}}{S_{y}^{2}}\right) \eta_{i}^{2}-2 R_{(i)}\left(\rho \frac{S_{x}}{S_{y}}\right) \eta_{i}\right\}^{-1} \times 100, \quad(i=1$ to 21$)$,

$\operatorname{PRE}\left(t_{C R(i)}, \bar{y}\right)=\left\{1+4 R_{(i)}^{2}\left(\frac{S_{x}^{2}}{S_{y}^{2}}\right) \eta_{i}^{2}-4 R_{(i)}\left(\rho \frac{S_{x}}{S_{y}}\right) \eta_{i}\right\}^{-1} \times 100, \quad(i=1$ to 21$)$ 
where

$$
\begin{aligned}
& \eta_{i}=1 \quad \text { for } \quad i=1 \quad \text { to } 5,18 \\
& \eta_{6}=\eta_{10}=\eta_{14}=\eta_{20}=\beta_{2(x)}, \quad \eta_{7}=\eta_{9}=\eta_{15}=\eta_{19}=C_{x}, \\
& \eta_{8}=\eta_{11}=\eta_{16}=\eta_{21}=\rho, \quad \eta_{12}=\eta_{13}=\eta_{17}=S_{x} ; \\
& R_{(1)}=\frac{\bar{Y}}{\bar{X}}, \quad R_{(2)}=\frac{\bar{Y}}{\bar{X}+C_{x}}, R_{(3)}=\frac{\bar{Y}}{\bar{X}+\beta_{2(x)}}, \\
& R_{(4)}=\frac{\bar{Y}}{\bar{X}+\rho}, \quad R_{(5)}=\frac{\bar{Y}}{\bar{X}+S_{x}}, \quad R_{(6)}=\frac{\bar{Y}}{\left(\beta_{2(x)} \bar{X}+C_{x}\right)}, \\
& R_{(7)}=\frac{\bar{Y}}{\left(\bar{X} C_{x}+\beta_{2(x)}\right)}, \quad R_{(8)}=\frac{\bar{Y}}{\left(\rho \bar{X}+C_{x}\right)}, \quad R_{(9)}=\frac{\bar{Y}}{\left(\bar{X} C_{x}+\rho\right)}, \\
& R_{(10)}=\frac{\bar{Y}}{\left(\bar{X} \beta_{2(x)}+\rho\right)}, \quad R_{(11)}=\frac{\bar{Y}}{\left(\rho \bar{X}+\beta_{2(x)}\right)}, \quad R_{(12)}=\frac{\bar{Y}}{\left(\bar{X} S_{x}+C_{x}\right)}, \\
& R_{(13)}=\frac{\bar{Y}}{\left(\bar{X} S_{x}+\beta_{2(x)}\right)}, \quad R_{(14)}=\frac{\bar{Y}}{\left(\bar{X} \beta_{2(x)}+S_{x}\right)}, \quad R_{(15)}=\frac{\bar{Y}}{\left(\bar{X} C_{x}+S_{x}\right)}, \\
& R_{(16)}=\frac{\bar{Y}}{\left(\rho \bar{X}+C_{x}\right)}, \quad R_{(17)}=\frac{\bar{Y}}{\left(\bar{X} S_{x}+\rho\right)}, \quad R_{(18)}=\frac{\bar{Y}}{(\bar{X}+X)}, \\
& R_{(19)}=\frac{\bar{Y}}{\left(\bar{X} C_{x}+X\right)}, \quad R_{(20)}=\frac{\bar{Y}}{\left(\bar{X} \beta_{2(x)}+X\right)}, \quad R_{(21)}=\frac{\bar{Y}}{(\bar{X} \rho+X)} .
\end{aligned}
$$

Findings are shown in Table 3. 
Table 3. PRE of $t_{R(i)}$ and $t_{C R(i)}(\mathrm{i}=1$ to 21$)$ with respect to $\bar{y}$

\begin{tabular}{|c|c|c|c|c|c|}
\hline $\begin{array}{c}\text { Estimator } \\
t_{R(i)}(\mathrm{i}=1 \text { to } 21)\end{array}$ & $\operatorname{PRE}(., \bar{y})$ & $\frac{k}{\theta_{(i)}}$ & $\begin{array}{c}\text { Estimator } \\
t_{C R(i)}(\mathrm{i}=1 \text { to } 21)\end{array}$ & $\operatorname{PRE}(., \bar{y})$ & $\frac{k}{\theta_{(i)}}$ \\
\hline$t_{R(1)}$ & $226.58^{*}$ & 1.70 & $t_{C R(1)}$ & 286.73 & 1.70 \\
\hline$t_{R(2)}$ & 225.21 & 1.71 & $t_{C R(2)}$ & 288.59 & 1.71 \\
\hline$t_{R(3)}$ & 210.73 & 1.88 & $t_{C R(3)}$ & 302.62 & 1.88 \\
\hline$t_{R(4)}$ & 226.02 & 1.70 & $t_{C R(4)}$ & 287.50 & 1.70 \\
\hline$t_{R(5)}$ & 131.02 & 5.13 & $t_{C R(5)}$ & 173.10 & 5.13 \\
\hline$t_{R(6)}$ & 226.52 & 1.70 & $t_{C R(6)}$ & 286.80 & 1.70 \\
\hline$t_{R(7)}$ & 218.29 & 1.79 & $t_{C R(7)}$ & 296.64 & 1.79 \\
\hline$t_{R(8)}$ & 224.91 & 1.72 & $t_{C R(8)}$ & 288.98 & 1.72 \\
\hline$t_{R(9)}$ & 226.30 & 1.70 & $t_{C R(9)}$ & 287.11 & 1.70 \\
\hline$t_{R(10)}$ & 226.56 & 1.70 & $t_{C R(10)}$ & 286.76 & 1.70 \\
\hline$t_{R(11)}$ & 207.69 & 1.92 & $t_{C R(11)}$ & $304.08^{*}$ & 1.92 \\
\hline$t_{R(12)}$ & 226.57 & 1.70 & $t_{C R(12)}$ & 286.73 & 1.70 \\
\hline$t_{R(13)}$ & 226.54 & 1.70 & $t_{C R(13)}$ & 286.78 & 1.70 \\
\hline$t_{R(14)}$ & 214.46 & 1.83 & $t_{C R(14)}$ & 300.07 & 1.83 \\
\hline$t_{R(15)}$ & 150.96 & 3.40 & $t_{C R(15)}$ & 226.58 & 3.40 \\
\hline$t_{R(16)}$ & 126.47 & 5.88 & $t_{C R(16)}$ & 161.21 & 5.88 \\
\hline$t_{R(17)}$ & $226.58^{*}$ & 1.70 & $t_{C R(17)}$ & 286.73 & 1.70 \\
\hline$t_{R(18)}$ & 100.74 & 181.76 & $t_{C R(18)}$ & 101.49 & 181.76 \\
\hline$t_{R(19)}$ & 101.49 & 90.93 & $t_{C R(19)}$ & 103.01 & 90.93 \\
\hline$t_{R(20)}$ & 117.05 & 8.70 & $t_{C R(20)}$ & 137.66 & 8.70 \\
\hline$t_{R(21)}$ & 100.61 & 221.28 & $t_{C R(21)}$ & 101.22 & 221.28 \\
\hline
\end{tabular}

*Indicates the largest PRE

It is observed from Table 3 that the proposed chain ratio-type estimators $t_{C R(i)}, \quad(\mathrm{i}=1$ to 21$)$ are better than the corresponding ratio-type estimator $t_{R(i)}$, (i=1 to 21) with substantial gain in efficiency. Largest gain in efficiency is observed by using over usual unbiased estimator $\bar{y}$. It is also noted that the estimator $t_{C R(11)}$ is the best followed by $t_{C R(14)}$ among all the estimators listed in Tables 1 and 2.

\section{Acknowledgment}

Authors are thankful to the Editor in Chief Professor Hamid Reza Navvabpour 
and the two learned referees for their valuable suggestions regarding improvement of the earlier draft of the paper.

\section{References}

Kadilar, C. and Cingi, H. (2003). Ratio estimators in stratified random sampling, Biometrical Journal 45, 218-225.

Kadilar, C. and Cingi, H. (2006). An improvement in estimating the population mean by using the correlation coefficient. Hacette. Jour. Math. Statist. 35, 103-109.

Lohr, S.L. (1999). Sampling: Design and Analysis. Duxbury Press.

Mohanty, S.and Das, M.N. (1971). Use of transformation in Sampling, Jour. Ind. Soc. Agri. Statist. 23, 83-87.

Rao, T.J. (1991). On certain methods of improving ratio and regression estimators, Commu. Statist. Theo. Metho. 20, 3325-3340.

Singh H.P. and Tailor R. (2003). Use of known correlation coefficient in estimating the finite population mean. Statist. Trans. 6, 555-560.

Singh, H.P. and Agnihotri, N. (2008). A general procedure of estimating population mean using auxiliary information in sample surveys. Statist. Trans.-new series 8, 71-87.

Singh, H.P., Tailor, R., Tailor, R. and Kakran, M.S. (2004). An improved estimator of population means using power transformation. Jour. Ind. Soc. Agri. Statist. 58, 223-230.

Sisodia, B.V.S. and Dwivedi, V.K. (1981). A modified ratio estimator using coefficient of an auxiliary variable. Jour. Ind. Soc. Agri. Statist. 33, 13-18.

Srivastava, S.K. (1967). An estimator using auxiliary information in sample surveys. Cal. Statist. Assoc. Bull. 16, 121-132.

Srivenkataramana, T. (1978). Change of origin and scale in ratio and difference methods of estimation. The Canad. Jour. Statist. 6, 79-86.

Srivenkataramana, T. (1980). A dual to ratio estimator in sample surveys. Biometrika 67, 199-204.

Tripathi, T.P. and Singh, H.P. (1992). A class of unbiased product type estimators for the mean suitable for positive and negative correlation situations. Commun. Statist. Theo. Metho. 21, 507-518.

Upadhyaya, L.N. and Singh, H.P. (1999). Use of transformed auxiliary variable in estimating the finite population means, Biometrical Journal 41, 627-636. 
Wolter, K.M. (1985). Introduction to Variance Estimation. Springer-Verlag.

\section{Housila P. Singh}

School of Studies in Statistics,

Vikram University,

Ujjain, 456010,

India.

email: hpsujn@gmail.com

email: anjanarathour01@gmail.com

\section{Anjana Rathore}

School of Studies in Statistics,

Vikram University,

Ujjain, 456010,

India. 